\title{
Simultaneous Total Laparoscopic Hysterectomy during Deep Inferior Epigastric Artery Perforator Flap Breast Reconstruction and Contralateral Superficial Inferior Epigastric Artery Flap Breast Augmentation
}

\author{
Toshihiko Satake, MD ${ }^{1}$ Masakazu Kitagawa, MD ${ }^{2}$ Ryunosuke Ninomiya, MD ${ }^{1} \quad$ Koichi Hirotomi, MD ${ }^{1}$ \\ Yasushi Yamamoto, MD ${ }^{3}$ Shinji Kobayashi, MD ${ }^{4}$ Hideya Sakakibara, MD ${ }^{2}$ jiro Maegawa, MD ${ }^{5}$
}

\footnotetext{
${ }^{1}$ Department of Plastic and Reconstructive Surgery, Yokohama City University Medical Center, Yokohama, Kanagawa, Japan

2 Department of Gynecology, Yokohama City University Medical Center, Yokohama, Kanagawa, Japan

${ }^{3}$ Department of Plastic and Reconstructive Surgery, Yokohama Rosai Hospital, Yokohama, Kanagawa, Japan

${ }^{4}$ Department of Plastic and Reconstructive Surgery, Kanagawa Children's Medical Center, Yokohama, Kanagawa, Japan

${ }^{5}$ Department of Plastic and Reconstructive Surgery, Yokohama City University Hospital, Yokohama, Kanagawa, Japan
}

J Reconstr Microsurg Open 2016;1:67-70.
Address for correspondence Toshihiko Satake, MD, Department of Plastic and Reconstructive Surgery, Yokohama City University Medical Center, 4-57 Urafune-cho, Minami-ku, Yokohama, Kanagawa 232-0024, Japan (e-mail: toshi@yokohama-cu.ac.jp).

At the first examination of our patient, she complained of heavy menstrual bleeding, prolonged menstrual periods, and frequent urination; the physical examination identified an irregular, hard, large intra-abdominal mass. After consultation with the gynecological department and a computed tomography (CT) scan, she was diagnosed with large uterine fibroids, which spanned from the pelvic cavity to the upper abdomen (-Fig. 2). She had no previous abdominal surgeries, and we estimated that there was enough abdominal fat volume to perform breast procedures using lower abdominal flaps.

Because of her symptoms and examination findings, a gynecological procedure (myomectomy or hysterectomy) was indicated. Additionally, under these circumstances, we thought that it would be difficult to achieve a tension-free wound closure of the donor site after the flap harvest. It seemed more effective to reduce or remove the uterine fibroids prior to reconstruction. The patient also desired a simultaneous hysterectomy following the breast reconstruction. Because the preoperative assessment in patients with large uterine fibroids is important to avoid significant lifethreatening complications such as deep venous thrombosis (DVT) and pulmonary thromboembolism (PE), we assessed her D-dimer levels (normal) and found no thromboembolism in her lower extremities by ultrasonography.

Copyright $\odot 2016$ by Thieme Medical Publishers, Inc., 333 Seventh Avenue, New York, NY 10001, USA. Tel: +1(212) 584-4662.
DOI http://dx.doi.org/ 10.1055/s-0036-1584220. ISSN 2377-0813. accepted after revision

April 3, 2016

published online

May 20, 2016 


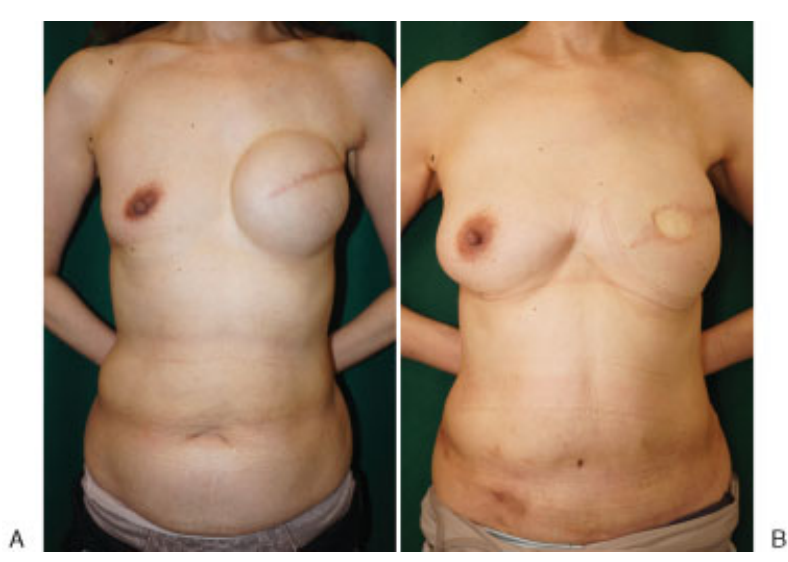

Fig. 1 (A) A 52-year-old multiparous woman (body mass index, 20.5 $\mathrm{kg} / \mathrm{m}^{2}$; bra cup size, A) presented with a delayed tissue expansion after a left mastectomy. She had an irregular, hard, large intra-abdominal mass due to uterine fibroids and complained of several symptoms. She had not undergone any prior abdominal surgeries. (B) The early postoperative result showed a reconstructed left breast with a small skin paddle, an augmented right breast, and significant improvement in her barrel abdomen.

\section{Surgical Procedures}

After removal of the left tissue expander and preparation of both recipient sites, we elevated a right deep inferior epigastric artery perforator (DIEP) flap based on three medial perforators for the left breast reconstruction and a left superficial inferior epigastric artery (SIEA) flap for the right breast augmentation with the patient in a supine position. A microsurgical anastomosis was first performed between the DIEP flap pedicle and the left internal mammary vessel. The SIEA flap pedicle was then anastomosed to the distal stump of the right deep inferior epigastric vessel. The de-epithelized SIEA flap was subcutaneously placed beneath the right mammary glands through the midline, and the DIEP flap was temporarily fixed to the left chest.

After a two-layer closure of the right incised rectus anterior fascia, the gynecologic surgical team began to perform a total hysterectomy with laparoscopic approach in the low lithotomy position with an intent to decrease postoperative pain and adhesion of bowels. The main trocar was inserted at the linea alba, $3 \mathrm{~cm}$ above the umbilicus to preserve the umbilical shape and circulation; three small trocars were placed just lateral to both outlines of the rectus muscles and the suprapubic linea alba ( $\mathbf{- F i g . ~ 3 A}$ ). Through these trocars, the uterus was dissected with a Harmonic scalpel (Ethicon, Cincinnati, $\mathrm{OH}$ ) and then divided into several small pieces for removal through the vaginal cavity (-Fig. 3B).

After a Penrose drain was placed into the Douglas cavum, the patient was again placed in the supine position. The lower abdominal skin was easily closed with two suture layers without any tension (-Fig. 4). At the same time, the left breast was reconstructed using the de-epithelized DIEP flap with a small skin paddle. The amount of blood loss was $400 \mathrm{~mL}$ and hysterectomy weight was $1,143 \mathrm{~g}$. Her postoperative course was uneventful (- Fig. 1B).

\section{Discussion}

Uterine fibroids are typically asymptomatic. ${ }^{1}$ However, as they enlarge in size, they may cause other symptoms and can also compress the surrounding anatomic structures. ${ }^{2}$ DVTs caused by pelvic vein compression are more complex with larger tumors, particularly in cases with uterine weights $>1,000 \mathrm{~g}^{3}$ Shah et al reported on a patient whose legs

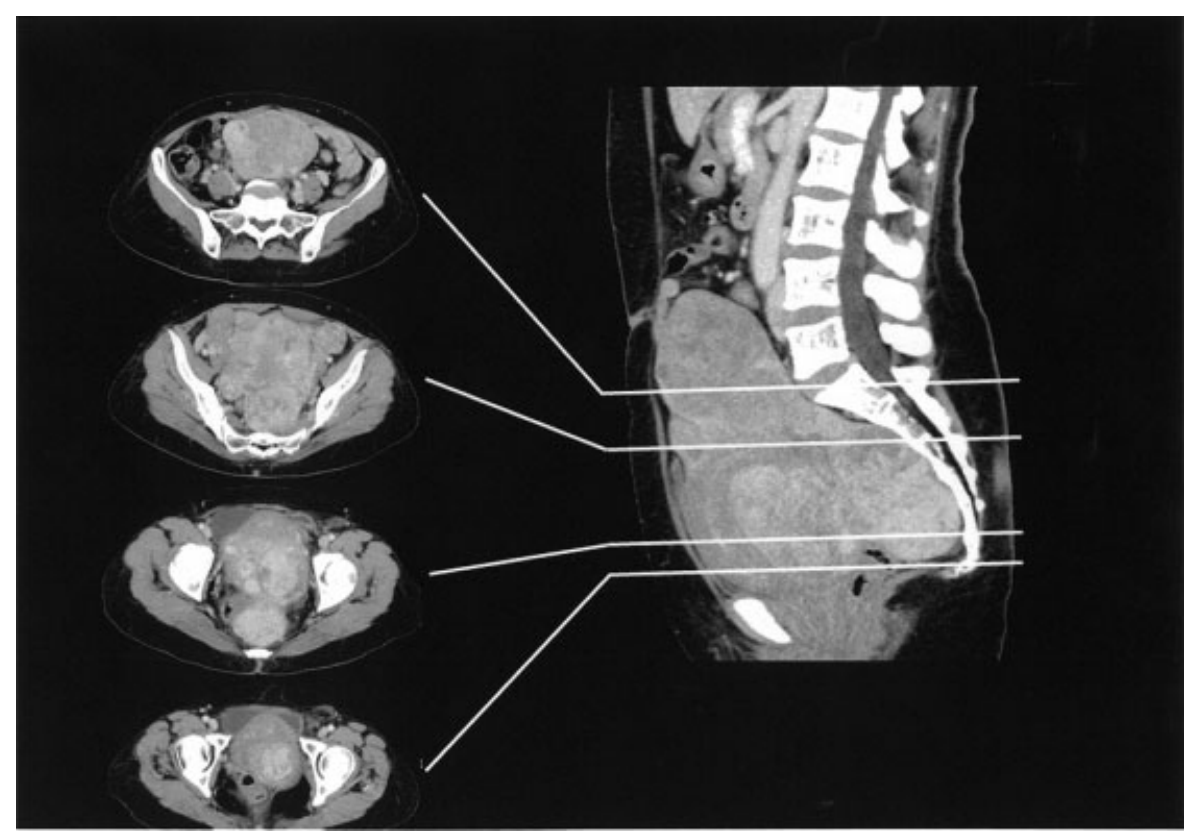

Fig. 2 Preoperative computed tomography showed an irregular large mass in continuity with the uterus, which is a typical finding with uterine fibroids; this extended from the pelvic cavity to the upper abdomen. She had enough abdominal fat volume to produce both lower abdominal flaps. 


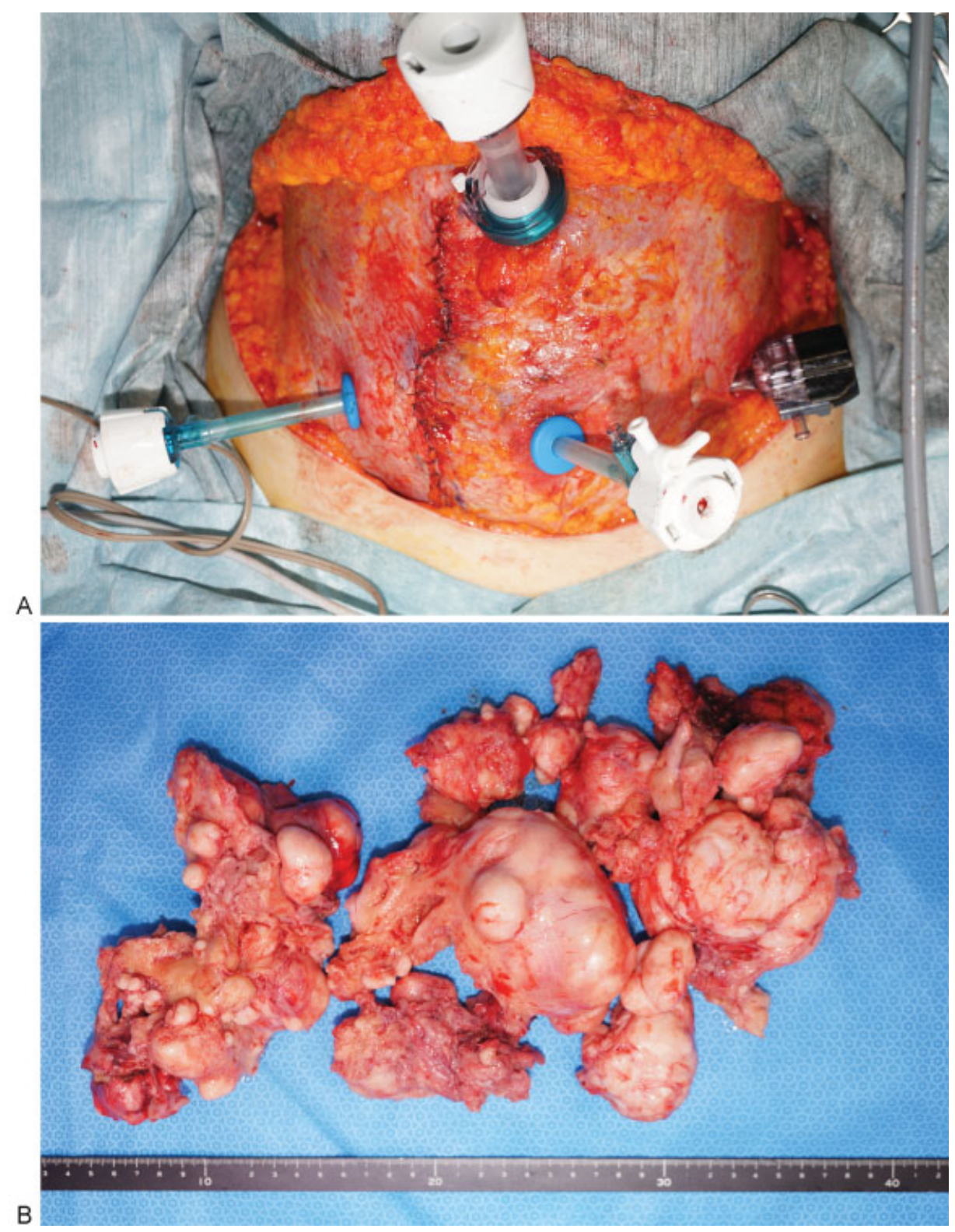

Fig. 3 (A) The main large trocar (showed superiorly in this picture) was inserted the linea alba $3 \mathrm{~cm}$ above the umbilicus to preserve the umbilical shape and circulation; three smaller trocars were placed just lateral to both outlines of the rectus muscles and suprapubic linea alba. (B) Under the laparoscope, the uterus was dissected and divided into several small pieces in the pelvic cavity, and then removed in pieces through the vagina. The hysterectomy weight was $1,143 \mathrm{~g}$.

were swollen because of compression by an enlarged uterus immediately after a DIEP flap breast reconstruction. ${ }^{4}$ Tension in a donor-site closure after lower abdominal flap breast reconstruction also increased intra-abdominal pressure. ${ }^{5}$ We should pay attention to severe complications such as DVT and PE, particularly with large uterine fibroids.

Determining whether to perform a gynecological surgery for large uterine fibroids before, simultaneously, or after a lower abdominal flap breast reconstruction is controversial. With laparoscopic surgery after breast reconstruction, care should be taken to recognize any anatomic translocations, including the umbilicus. ${ }^{6,7}$ Previous studies showed increased donor-site complications in abdominal-based breast reconstruction patients who had prior abdominal surgeries. ${ }^{8,9}$ Laparoscopic surgery before reconstruction has the potential risk of deep inferior epigastric vessel injury, ${ }^{10}$ and can leave umbilical scars. This scarring may cause decreased umbilical blood circulation when the neighboring skin is incised during abdominal-based breast reconstruction.

There have been no reports detailing a simultaneous gynecological procedure during a lower abdominal flap breast reconstruction. One of the disadvantages of a laparoscopic approach was the challenge of maintaining insufflation with the thin abdominal wall after the flap harvest. Besides, intraoperative position change was necessary twice for this procedure. However, simultaneous procedures are feasible and can have many advantages. The patient can undergo simultaneous breast reconstruction and TLH. There 


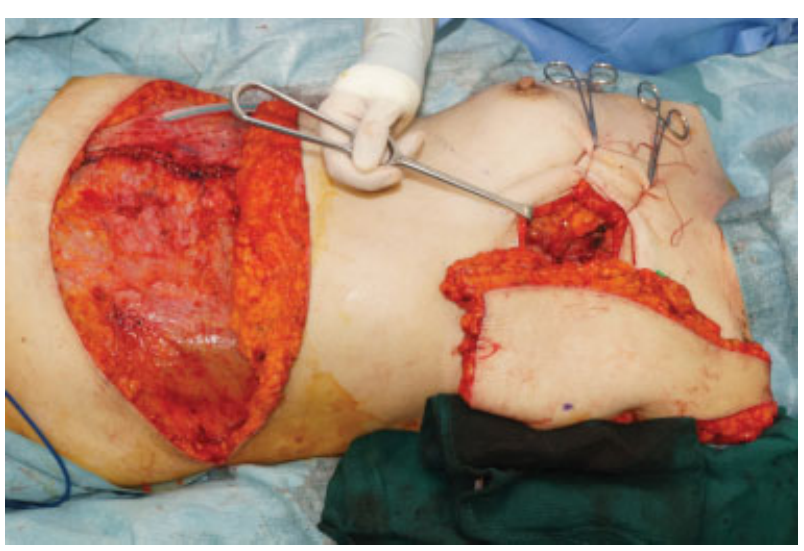

Fig. 4 After a Penrose drain was placed into the Douglas cavum, the lower abdominal skin was easily closed with two suture layers without any tension. The left breast was reconstructed using the DIEP flap. The head of the muscle retractor indicated the SIEA flap, which was placed under the right mammary glands.

are no additional scars except with the flap harvesting; moreover, there is no risk of injury to the nutrient vessels and subcutaneous vascular network of the abdominal flap because the flap harvest is performed first. Finally, these procedures allow the surgeons to perform tension-free donor-site closures, which lead to less postoperative pain and complications.

\section{Conflict of Interest \\ None.}

\section{References}

1 National Women's Health Information Center, U.S. Department of Health and Human Services. ePublications: uterine fibroids fact sheet. Available at: http://www.womenshealth.gov/publications/our-publications/fact-sheet/uterine-fibroids.html. Accessed September 24, 2015

2 Khademvatani K, Rezaei Y, Kerachian A, Seyyed-Mohammadzad MH, Eskandari R, Rostamzadeh A. Acute pulmonary embolism caused by enlarged uterine leiomyoma: a rare presentation. Am J Case Rep 2014;15:300-303

3 Shiota M, Kotani Y, Umemoto M, et al. Deep-vein thrombosis is associated with large uterine fibroids. Tohoku J Exp Med 2011; 224(2):87-89

4 Shah AK, Sala E, Staiano JJ. If the scan fits-evacuate!. J Plast Reconstr Aesthet Surg 2009;62(4):554-555

5 Losken A, Carlson GW, Tyrone JW, et al. The significance of intraabdominal compartment pressure after free versus pedicled TRAM flap breast reconstruction. Plast Reconstr Surg 2005; 115(1):261-263

6 Awtrey CS, Abu-Rustum NR, Disa JJ, et al. Laparoscopic bilateral salpingo-oophorectomy in breast cancer patients after transverse rectus abdominus myocutaneous flap reconstructive surgery. Gynecol Oncol 2005;99(3):720-725

7 Muller CY, Coleman RL, Adams WP Jr. Laparoscopy in patients following transverse rectus abdominis myocutaneous flap reconstruction. Obstet Gynecol 2000;96(1):132-135

8 Parrett BM, Caterson SA, Tobias AM, Lee BT. DIEP flaps in women with abdominal scars: are complication rates affected? Plast Reconstr Surg 2008;121(5):1527-1531

9 Nahabedian MY, Manson PN. Contour abnormalities of the abdomen after transverse rectus abdominis muscle flap breast reconstruction: a multifactorial analysis. Plast Reconstr Surg 2002; 109(1):81-87, discussion 88-90

10 Hurd WW, Pearl ML, DeLancey JO, Quint EH, Garnett B, Bude RO. Laparoscopic injury of abdominal wall blood vessels: a report of three cases. Obstet Gynecol 1993;82(4, Pt 2, Suppl):673-676 